\title{
Prediction of Calibration Parameters of the Oxygen Saturation Estimation Equation by Optical Recording on Smartphones
}

Prediction Parameters $\% \mathrm{SpO}_{2}$ by Optical Recording

\section{DANIEL CABALLEROS-TEJADA*}

Carrera de Electrónica e Instrumentación, Universidad de las Fuerzas Armadas-ESPE, Ecuador

decaballeros@espe.edu.ec

DAVID RIVAS-LALALEO

Departamento de Eléctrica y Electrónica, Universidad de las Fuerzas Armadas ESPE, Ecuador

drrivas@espe.edu.ec

This paper demostrate the calculate for calibration parameters of the Percent Oxygen Saturation $\left(\% \mathrm{SpO}_{2}\right)$ estimation equation by means of a multiple linear regression type supervised machine learning algorithm with the objective of being able to use a smartphone as an oximeter, for this a training and test data set composed of predictor variables representing the characteristics of the smartphones used in this development is used, in addition to the statistical parameters extracted from two thousand videos, predicting values of $\% \mathrm{SpO}_{2}$ with an error of less than $2 \%$ with respect to a standard oximeter.

CCS CONCEPTS - Computing methodologies $\rightarrow$ Machine learning $\rightarrow$ Learning paradigms $\rightarrow$ Supervised learning $\rightarrow$ Supervised learning by regression

Additional Keywords and Phrases: Oxygen Saturation, machine learning, datasets, video, smarthphone

\section{ACM Reference Format:}

Daniel Caballeros-Tejada and David Rivas-Lalaleo. 2021. Prediction of Calibration Parameters of the Oxygen Saturation Estimation Equation by Optical Recording on Smartphones. In IEAI '21: 2nd International conference on Industrial Engineering and Artificial Intelligence, April 02-04, 2021, Osaka, Japon. ACM, New York, NY, USA, 10 pages.

\footnotetext{
* Place the footnote text for the author (if applicable) here.

Permission to make digital or hard copies of all or part of this work for personal or classroom use is granted without fee provided that copies are not made or distributed for profit or commercial advantage and that copies bear this notice and the full citation on the first page. Copyrights for components of this work owned by others than ACM must be honored. Abstracting with credit is permitted. To copy otherwise, or republish, to post on servers or to redistribute to lists, requires prior specific permission and/or a fee. Request permissions from permissions@acm.org. (C) 2021 Association for Computing Machinery. Manuscript submitted to ACM
} 


\section{INTRODUCTION}

The pulse oximeter was introduced in 1984 by scientist Takuo Aoyagi [1], a biomedical device that has helped save millions of lives by measuring heart rate and oxygen saturation percentage [2]. This equipment is based on the physical principle that uses red and infrared light to measure oxygen levels in blood through hemoglobin, an oxygen-carrying protein [9], which has a measurable variation that links oxygenated blood flowing through the arteries. After the massive worldwide commercialization of smartphones, software development companies and independent programmers create applications that use the sensors of the device [6] to provide functionalities that devices such as pulse oximeter, Jordan [7] in 2019 develops an application to diagnose hypoxia, and also analyzes the feasibility of using a smartphone as a medical device. The World Health Organization (WHO) since 2020 [4] indicates that Covid-19 is an infectious disease caused by coronavirus, which affects the respiratory system, one in five patients develops severe disease with respiratory distress. Severe Acute Respiratory Infection (ARI) presents with clinical symptoms such as: central cyanosis or Percent oxygen saturation $\left(\% \mathrm{SpO}_{2}\right.$ ) less than $90 \%$. Patients with $\mathrm{ARI}$ requiring oxygen therapy should achieve a $\% \mathrm{SpO}_{2}$ greater than $90 \%$, in pregnant women the $\% \mathrm{SpO}_{2}$ should be between 92 to $95 \%$, for children greater than 94\% [3]. Studies conducted by the research group of the Clinic at Thomas Jefferson University [12] refer to the indispensable use of the pulse oximeter as a medical tool for out-of-hospital patients infected or with symptoms of Covid-19, since the oxygen saturation value of $90 \%$ between the eighth and twelfth day is an urgent indicator for oxygen therapy [4]. Patients who are treated at home, since they do not have biomedical equipment, it is impossible for them to inform the treating physician or health system with concrete and accurate values of vital signs in the evolution of the disease [11].

This article is structured as follows: it begins with an introduction on the subject to be addressed, followed by a methodology in which the theoretical foundations used in this work are described, then, in the experimentation, the experimental design developed is described, from which the results section is derived, where the most relevant data obtained are expressed, to end with the conclusions section.

\section{METHODOLOGY}

The percentage of oxygen saturation can be estimated using equation 1 [8] [5] [19] [14] [10], for this the optical recording methodology is applied with the back camera of smartphones with the incidence of a light source such as the flash of the camera on the finger of the person, as it allows capturing the incident heart rate, similar to a pulse oximeter. The smartphone makes a video clip of time $t$ to process it and extract statistical data that allow estimating the $\% \mathrm{SpO}_{2}$.

$$
S p O_{2}=A-B \frac{\left(A C_{R E D} / D C_{R E D}\right)}{\left({ }^{A} C_{B L U E} / D C_{B L U E}\right)}
$$

The parameters of equation 1 are defined as: $A C_{R E D}$ corresponds to the calculation of the standard deviation of the signal obtained from the standard deviation in red, $A C_{B L U E}$ corresponds to the calculation of the standard deviation of the signal obtained from the standard deviation in the color blue, $D C_{R E D}$ corresponds to the calculation of the average signal obtained from the average in red, $D C_{B L U E}$ corresponds to the calculation of the average of the signal obtained from the average in the color blue, $A$ y $B$ parameters that adjust the equation to calculate an estimate of $\% \mathrm{SpO}_{2}$. 
The values of $A C_{R E D}, A C_{B L U E}, D C_{R E D}$ and $D C_{B L U E}$ are obtained by processing the video clip of $\mathrm{t}$ seconds [8]. Transforming the video clip into 3 matrices composed by the amplitudes of the pixels in RGB, in these matrices is contained the cardiac pulse signal, this signal is directly proportional in intensity with the incidence of light (ambient and effect of the flash on) at the time of recording the video. To have a homogeneous composition of the signal in each frame, to reduce the deviations or noise resulting from the location of the flash and the ambient light that directly affects the quality of the sample and to facilitate the processing of the signals, the central area of the frame is cut into a matrix of $64 x 64$ pixels, generating 3 matrices of dimensions $64 x 64 x n$ Frames. By default, smartphones record at 30 frames per second, so the resulting matrices will be $64 x 64 x$ ( $30 * t$ [second]). The statistical parameters needed for the oxygen saturation percentage estimation equation are obtained by calculating the average and standard deviation of each $64 x 64 R G B$ matrix.

\subsection{Signal processing}

The cardiac pulse signal extracted from the video clip, which in turn contains the oxygen saturation based on the physiological principle that the human body performs to oxygenate the blood by transporting oxygenated hemoglobin, through the variation of the average and standard deviation in the 3 matrices composed of the colors Red and Blue allow estimating the percentage of oxygen saturation. To improve the quality of biomedical signals and reduce the incidence of factors outside the human body, the signals are processed using the following methods: Initial n-frame clipping: Reduces signal saturation caused by initial flash firing, common in some smartphone models. Low-pass Filter: Reduce electrical noise or typical hand vibration when recording video [16]. Spatial-Temporal Filter: Improve signal quality by choosing a threedimensional window of values that are averaged together, the greatest predominance for filtering is in the temporal filter with respect to the spatial [15] [18].

\subsection{Tunning Parameters}

Tunning parameters of equation $1 A$ and $B$ should be found experimentally [17] [13] using estimation methods according to $n$ number of videos, these values depend on the characteristics of the subject who recorded the video as well as the physical characteristics of the smartphone such as video quality and camera resolution. These values must be contrasted to quantify the calculated values with respect to a clinical pulse oximeter as a standard element, so the variables of $A$ and $B$ must generate an error of less than $2 \%$ to consider the robustness of the algorithm to predict the percentage of oxygen saturation.

\subsection{Machine Learning Algorithm}

Simple linear regression is a supervised learning model that is directly related to equation 1 , since it is an estimation model that allows obtaining alpha and beta values to predict an output value from an input value $x$ as indicated in equation 2. To improve the prediction result, Multiple Linear Regression can be used, decreasing the uncertainty since it uses $\mathrm{n}$ number of predictor variables $x n$, the representation of the model is shown in equation 3.

$$
\begin{aligned}
& y_{\text {model }}=\alpha+\beta(x)+\varepsilon \\
& y_{\text {model }}=\alpha+\beta_{1}\left(x_{1}\right)+\beta_{2}\left(x_{2}\right)+\cdots++\beta_{n}\left(x_{n}\right)+\varepsilon
\end{aligned}
$$


To: $y_{\text {model }}$ si value predicted by linear regression, $\alpha$ Independent term. Expected value if $x=0, \beta$ Coefficient of simple linear regression, $x$ Predictor value. Expected value if $x_{n}=0, \beta_{n}$ Coefficients of the multiple linear regression, $x_{n}$ Predictor values, $\varepsilon$ Observation error due to uncontrolled variables.

The predictor model is evaluated against the criteria for testing a prediction: Total Variability $(V T)$ : It is the sum of the explained and unexplained variability, calculated with equation 4, Explained Variability $(V E)$ : It is the sum of the squares of the regression expressed in equation 5 , Unexplained Variability $(V N E)$ : It is the sum of the residual squares expressed in equation 6, Coefficient of Determination $\left(R^{2}\right)$ : It is defined as the proportion of variability of the dependent variable that is explained by the regression in equation 7 , it is considered a good model when the coefficient of determination is greater than 0.8 with a maximum of 1 , and the model is discarded when it approaches the minimum value of 0 [20]:

$$
\begin{aligned}
& V T=V E+V N E \\
& V E=\sum_{i=1}^{n}\left(\hat{y}_{i}-\bar{y}_{i}\right)^{2} \\
& V N E=\sum_{i=1}^{n}\left(y_{i}-\bar{y}_{i}\right)^{2} \\
& R^{2}=\frac{V E}{V T}
\end{aligned}
$$

\subsection{DataSet}

The model of a predictor algorithm is made from the creation of a dataset with correlated independent variables. It includes descriptive 21 posible predictor variables: $D C_{R}, D C_{G}, D C_{B}, A C_{R}, A C_{G}, A C_{B},\left(A C_{R} / D C_{R}\right) /\left(A C_{B} / D C_{B}\right)$,

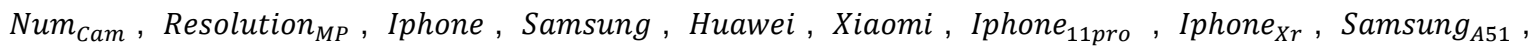
Samsung $_{\text {A10 }}$, Huawei $_{\text {P30lite }}$, Huawei $_{\text {P40lite }}$, Xiaomi $_{\text {Note8Pro }}$, Xiaomi $_{\text {Note9Pro }}$.

Multicollinearity between predictor variables is a criterion that helps to avoid the over description of a multiple linear model, it occurs when the predictor variables of the model have a significant correlation causing the variability of the estimated coefficient, this can be avoided by searching for pairs of predictor variables and eliminating one of them so that it does not affect the estimation model, using the VIF (Variance Inflation Factor) technique, the variables to be eliminated are determined, it is calculated from equation 8 . If the VIF is 1 , the variables are not correlated; if it is between 1 and 5 , the variables are moderately related and can form part of the predictive model; if the value is greater than 5 , the variables should be eliminated from the model [20].

$$
V I F=\frac{1}{1-R^{2}}
$$

\subsection{Video Database}

A database of 2000 videos obtained from the 4 popular smartphone brands (Iphone, Samsung, Huawei and Xiaomi), in turn subdivided into groups of 250 videos with 8 models (Iphone: 11 Pro and Xr; Samsung: A51 and A10; Huawei: P30Lite and P40Lite; Xiaomi: Note8pro and Note9pro) with their own characteristics such as the number of cameras and video recording resolution. The videos are approximately 1 minute long and are recorded by placing the index finger on the smartphone's rear camera with the flash on. By processing this database, the training and testing dataset is created to find a linear regression model to estimate $\% \mathrm{SpO} \mathrm{O}_{2}$. 


\section{EXPERIMENTATION}

\subsection{Signal processing}

After converting the video into the 3 RGB matrices of $64 x 64 x(30 * t$ [second]) of a video, the signal is extracted and the size is standardized to 1800 frames (60seconds) eliminating those that are in excess at the end, to avoid the initial saturation of the signal caused by the ignition of the flash in the recording, the initial $10 \%$ of frames (6seconds) is cut, this guarantees to have a homogeneous signal in amplitude, in figure 1 (a) it verifies in the signal $D C_{R E D}$ being this one the one of greater amplitude with respect to its similar ones. With the clipped signal, a low-pass filter is applied to eliminate the noise produced by the hand vibration at the moment of recording the video. The cutoff frequency and the order of the filter are selected using a filtered signal that does not attenuate the amplitude or phase shift excessively with respect to the original signal. Using an algorithm that increases the values of $\mathrm{Order}=\left[\begin{array}{l}9 \text { to } 12\end{array}\right]$ and $f c=\left[\begin{array}{ll}0.8 \text { to } 1.4\end{array}\right] \mathrm{Hz}$ with a constant sampling rate of $3 \mathrm{~Hz}$. A portion of the signal is plotted to verify which signal meets the selection criteria, figure 1 (b) represents this process, selecting $\operatorname{Order}=12$ and $f c=1 \mathrm{~Hz}$. To smooth the signal a median filter in 3 dimensions is used, the algorithm varies the size of the kernel so that in its spatial component $(x, y)$ we maintain an odd increasing matrix from 3 to 7 pixels, while the temporal component (z) matrix will be from 3 to 9 pixels. In figure 1 (c) verifies this process and considers as the selection criterion for the filter kernel, the attenuation of the signal with respect to the temporal component, the selected kernel is $[x=5, y=5, z=7]$. The figure 1 (d) shows a window of the signal to verify the action of the filters on the $D C_{R E D}$ and $A C_{R E D}$ signals. Reducing greatly the signal noise, without attenuating and avoiding a deface with respect to the original signals (black).

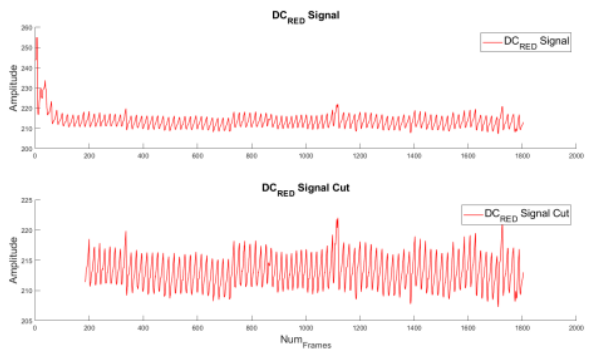

(a)

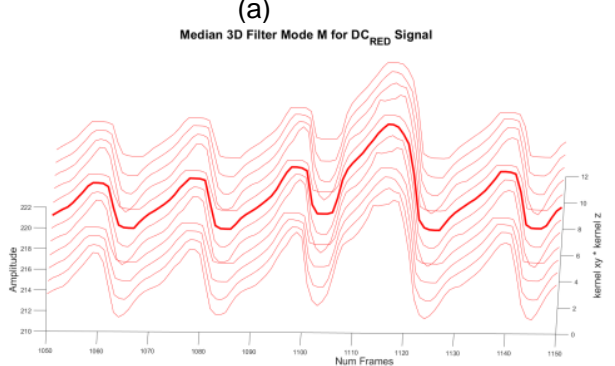

(c)

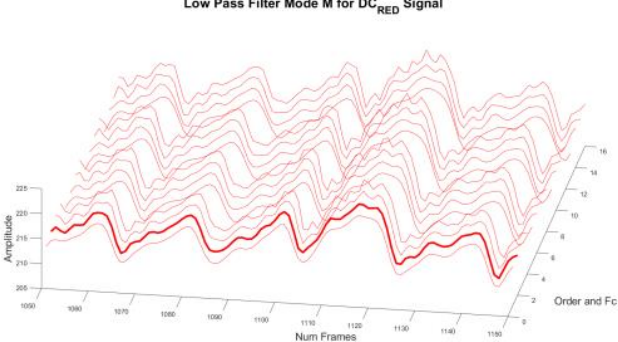

(b)

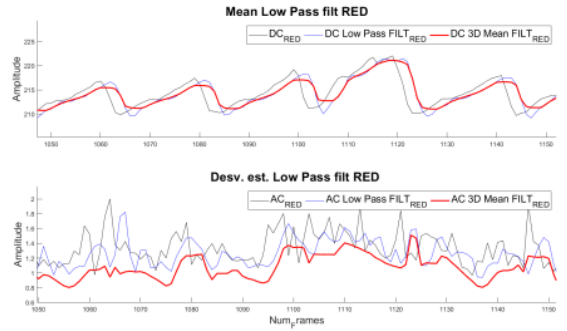

(d)

Figure 1: Signal Process: (a) Original Signal, (b) Low Pass Filtering, (c) Space Temp Filtering, (d) Original Vs Proceses Signal.

\subsection{Machine Learning}

The dataset used for training and testing the predictor model is created from the 2000 videos, the columns that compose it are: Brand, Model, Number of Cameras, Resolution in MegaPixels, $\% \mathrm{SpO}_{2}$ Measured with an 
Oximeter, Heart Rate Measured with an Oximeter, Average of the filtered $D C_{R}$ signal, Average of the filtered $D C_{G}$ signal, Average of the filtered $D C_{B}$ signal, Standard Deviation of the filtered $A C_{R}$ signal, Standard Deviation of the filtered $A C_{G}$ signal, Standard Deviation of the filtered $A C_{B}$ signal and the resulting value of $\left(A C_{R E D} / D C_{R E D}\right) /\left(A C_{B L U E} / D C_{B L U E}\right)$. Figure 2 shows the statistical parameters of the dataset. In Figure 3 plots the statistical parameters of the videos as a function of $\% \mathrm{SpO}_{2}$, since they are linearly related, a simple or multiple linear regression can be applied to estimate $\% \mathrm{SpO}_{2}$. The columns Brand and Model contain character strings so they cannot be considered as predictor variables, by converting them into dummy variables they will be of type $x_{n}$ as in the equation 3 , so that all the columns can be predictor variables that enter the multiple linear regression to estimate the percentage of oxygen saturation, the columns $\% \mathrm{SpO}_{2}$ and Heart Rate measured with the standard oximeter are discarded as predictor variables, since $\% \mathrm{SpO}_{2}$ will serve as the variable to be predicted. The dataset is randomly divided in an $80 / 20$ ratio, $80 \%$ of the data will be used for training and $20 \%$ for linear regression validation. The prediction testing criteria are summarized in Table 1.

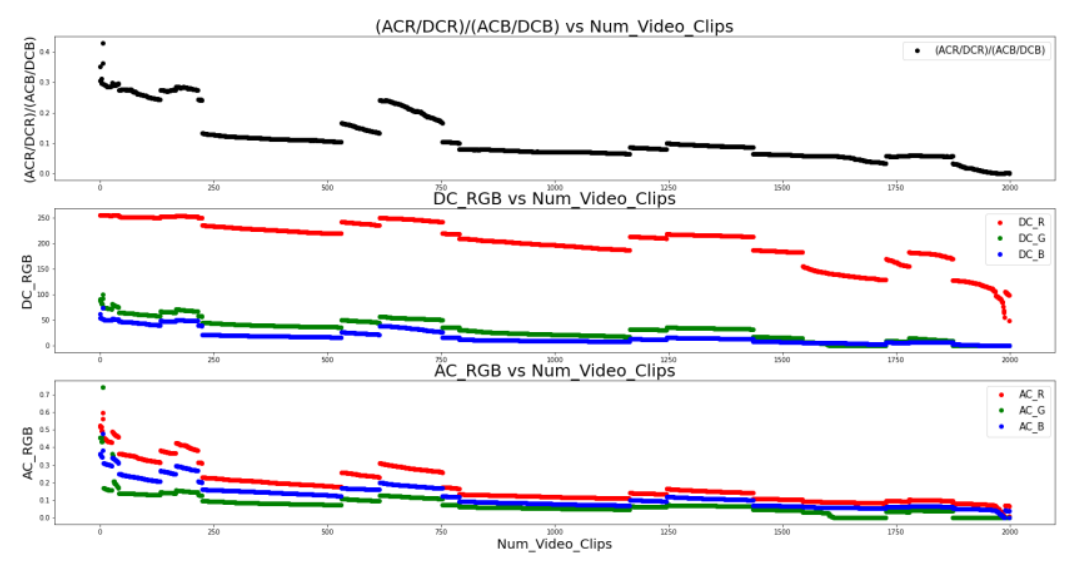

Figure 2: Statistical parameters of the DataSet as a function of number of video clips
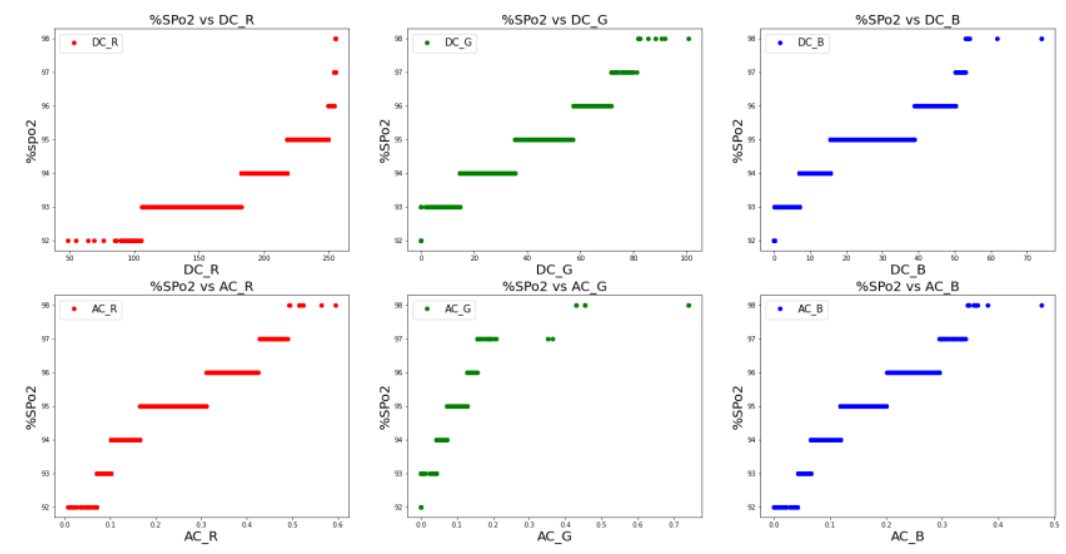

Figure 3: Statistical parameters of the DataSet sorted by $\% \mathrm{SpO}_{2}$ Measured with the Pulse Oximeter. 


\section{RESULTS}

The predictive models are shown in figure 4 , to determine the most efficient predictive model according to Table 1 , one of the most efficient using 7 predictor variables, figure 5 (b), from the statistical parameters has a fit coefficient of 0.9224 with an error of 0.29 , which means that $99.71 \%$ of the cases have a solution using multiple linear regression in equation 10 models the estimating equation for the $\% \mathrm{SpO}_{2}$. From the table we can also extract that the adjustment coefficient for linear models of more than 16 predictor variables does not have a significant improvement, on the contrary, simple linear regression could be used to facilitate the estimation of blood oxygen saturation by requiring only the processing of the signals to extract the statistical parameters in equation 9 models the estimating equation for the $\mathrm{SSpO}_{2}$. Multiple linear regression model with the highest coefficient of fit is the one that uses 21 predictor variables, model figure 5 (c). If a smartphone of a different brand and model were used, this would be a divergent value to the dataset used for training, decreasing the effectiveness of the predictor model in equation 11 models the estimating equation for the $\% \mathrm{SpO}_{2}$. Figure 4 shows the graph of the probabilistic estimators of the measured signal vs. the signals estimated with the different methods. Finally, the least efficient model has one predictor variable, with a fit coefficient 0.7961 , in Figure 5 (a).

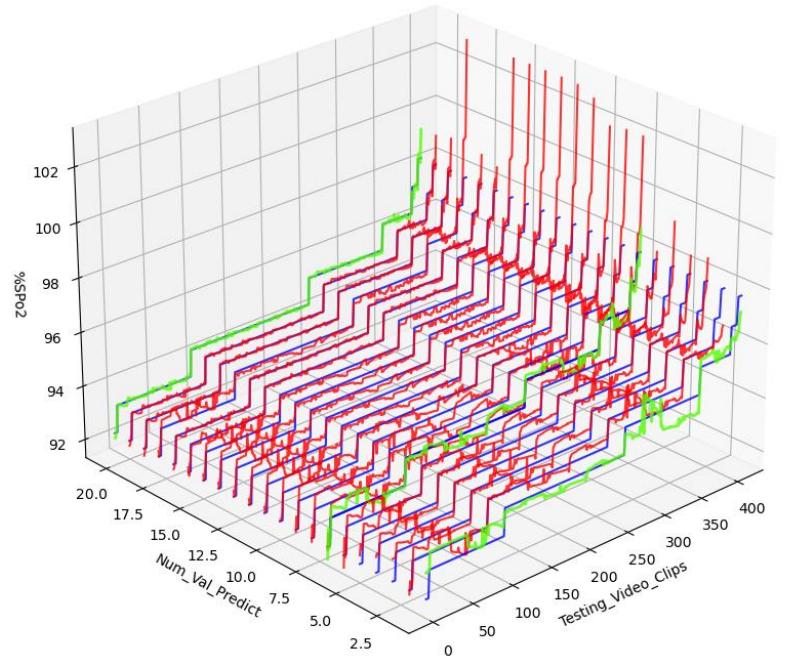

Figure 4: The predictive models Mode $\mathrm{M}$ as function of NumValPredictor.

Table 1: Criteria for testing a linear regression test for $\mathrm{n}$ predictor variables

\begin{tabular}{ccccccc}
\hline NumValPredictor & $R^{2}$ & $V N E$ & $V E$ & $V T$ & $V I F$ & ERROR\% \\
\hline 1 & 0.79618568 & 80.6082357 & 0.44613078 & 81.0543665 & 0.20381431 & 0.47307391 \\
3 & 0.89814123 & 39.0828843 & 0.31141589 & 39.3943002 & 0.10185876 & 0.33022320 \\
7 & 0.92241475 & 31.0732179 & 0.27906583 & 31.3522837 & 0.07758524 & 0.29591942 \\
15 & 0.94545076 & 52.6018575 & 0.36678550 & 52.9686430 & 0.05454923 & 0.38893674 \\
16 & 0.99236204 & 3.96451280 & 0.10082369 & 4.06533649 & 0.00763795 & 0.10691272 \\
17 & 0.99544094 & 2.96958182 & 0.08737211 & 3.05695393 & 0.00455905 & 0.09264876 \\
18 & 0.94545076 & 52.6018575 & 0.36820075 & 52.9700582 & 0.05454923 & 0.39043746 \\
19 & 0.99527160 & 3.33533748 & 0.09283557 & 3.42817305 & 0.00472839 & 0.09844217 \\
21 & 0.99544094 & 2.96958182 & 0.08782482 & 3.05740664 & 0.00455905 & 0.09312881 \\
\hline
\end{tabular}




$$
\begin{aligned}
& \mathrm{y}_{1 \text { var }_{\text {predict }}}=92.9368+12.3255 \frac{A C_{R} / D C_{R}}{A C_{B} / D C_{B}} \\
& \mathrm{y}_{7 \text { var }_{\text {predict }}}=90.6505-2.2092 \frac{A C_{R} / D C_{R}}{A C_{B} / D C_{B}}+ \\
& 0.0115\left(D C_{R}\right)-0.005\left(D C_{G}\right)-0.0263\left(D C_{B}\right)+ \\
& 5.6182\left(A C_{R}\right)+1.0988\left(A C_{G}\right)+9.1344\left(A C_{B}\right) \\
& \mathrm{y}_{21 \text { var }_{\text {predict }}}=90.8871-0.6829 \frac{A C_{R} / D C_{R}}{A C_{B} / D C_{B}}+0.0105\left(D C_{R}\right)-0.0088\left(D C_{G}\right)-0.0256\left(D C_{B}\right)- \\
& 0.6030\left(A C_{R}\right)+1.6555\left(A C_{G}\right)-12.609\left(A C_{B}\right)-0.0892\left(N u m_{C a m}\right)+0.0028\left(\operatorname{Res}_{M P}\right)+ \\
& 0.0472(\text { Iphone })+0.1470(\text { Samsung })-0.2375(\text { Huawei })+0.0431(\text { Xiaomi })-0.1438\left(\text { Iphone }_{11 \text { pro }}\right)+ \\
& 0.1911\left(\text { Iphone }_{X r}\right)+0.2243\left(\text { Samsung }_{A 51}\right)-0.0772\left(\text { Samsung }_{A 10}\right)-0.1858\left(\text { Huawei }_{P 30 l i t e}\right)+ \\
& 0.0517\left(\text { Huawei }_{\text {P4olite }}\right)+0.3010\left(\text { Xiaomi }_{\text {Note8Pro }}\right)-0.2579\left(\text { Xiaomi }_{\text {Note9Pro }}\right)
\end{aligned}
$$

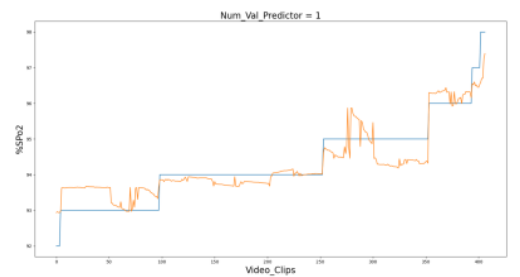

(a)

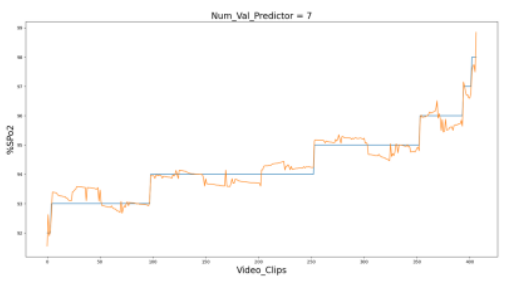

(b)

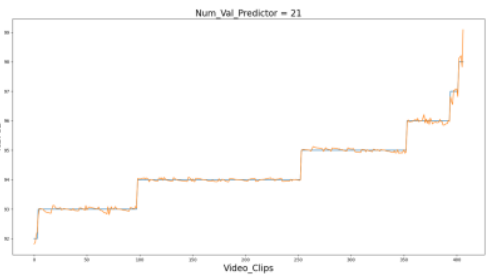

(c)

Figure 5: Predictor Model: (a) 1 variable, (b) 7 variables, (c) 21 variables.

\section{CONCLUSIONS}

The smartphones used in this development have provided insight into recurring features that can affect the signal. The flash at the beginning of the recording saturates the signal for approximately 3 seconds, during which time the camera's autofocus has problems that significantly affect the homogeneity of the signal. After 4 or 5 seconds the signal stabilizes, being necessary to cut $10 \%$ of the initial recording time, on the other hand, the incidence of external factors such as hand vibration at the time of recording the video and ambient light affect the quality of biomedical signals extracted from the video, therefore, the implementation of filters is important to eliminate frequencies higher than the heart rate. The strategy developed to find the filter modeling parameters was focused on a spatiotemporal analysis (Figures M Mode) in which the attenuation and phase shift generated by the filtering process on the original signal was evidenced, in this way a twelfth-order low-pass filter with a cutoff frequency of $1 \mathrm{~Hz}$ was selected, while in the spatiotemporal median filter kernel of [5x5x7] was selected. The quality of the obtained signals was substantial despite generating additional processing times. When generating the DataSet with all the videos, it was found as a recurrence that regardless of the smartphone model, the statistical parameters extracted from the signals with the highest numerical value are $D C_{R E D}$ and $A C_{R E D}$, furthermore they correlate directly with the measured value, thus providing a significant contribution to the prediction of the $\% \mathrm{SpO}_{2}$ value. The statistical parameters with the highest correlation with the measured $\% \mathrm{SpO}_{2}$ value according to the experimentation are $D C_{\text {GREEN }}$ and $A C_{G R E E N}$, which contribute significantly to the prediction of the $\% \mathrm{SpO}_{2}$ value, so multicollinearity is the ideal method to avoid that the prediction variables used are correlated with each other; after experimentation, an $R^{2}$ of was obtained 0.99544094 for the predictive 
model of 21 variables, it is the best model that fits the current DataSet, however when entering a new set of data from a different phone the model will be inaccurate, so it is recommended to use as predictive model the one of 7 variables despite having an $R^{2}$ of 0.92241475 with an error of $0.29591942 \%$.

The simple linear regression obtained in equation 9 manages to estimate the parameters of $A=\alpha=92.9368$ and $B=\beta=12.3255$ of equation 1 to calculate the $\% \mathrm{SpO}_{2}$, by getting unreliable estimates the multiple linear regression with predictor variables correlated with the measured $\% \mathrm{SpO}_{2}$ is developed, the parameters $\alpha$ and $\beta_{n}$ found for equations 10 and 11 allow to adjust the supervised learning algorithm to the training data set and validate the predictions on the test data set with a coefficient of determination higher than 0.8981 with 3 predictor variables, relative to 0.7961 for simple linear regression. Therefore, multiple linear regression with 3 predictor variables allow estimating reliable $\% \mathrm{SpO}_{2}$ values; consequently, if the algorithm uses 7 or more predictor variables, the predictions are considered highly reliable. Undoubtedly, the prediction algorithm based on multiple linear regressions is a very accurate alternative offered by supervised machine learning for estimating $\% \mathrm{SpO}_{2}$. This reliability is directly related to the number of predictor variables contained and the size of the data set. In this research, it is estimated that 2000 minutes of video recording and approximately 1900 minutes of preprocessing were used for the generation of the data set alone. Considering that 8 smartphone models were used, if new models were implemented, the video database would grow exponentially, requiring large storage space and processing power. Therefore, the methodology used for $\% \mathrm{SpO}_{2}$ estimation will be inviable with the wide range of smartphone models currently available in the market. Researches focused on $\% \mathrm{SpO}_{2}$ estimation such as the Convolutional Neural Networks method [5] achieves an error of $2.02 \%$ with respect to a pulse oximeter, this method can cover several smartphone models since it analyzes biomedical signals extracted from videos, on the contrary, if the smartphone is considered as a measuring instrument, the calibration curve can be obtained [8] [10] [10] [14] to obtain the $A$ and $B$ values indicated in equation 1 and calculate consistent estimates of $\% \mathrm{SpO}_{2}$. None of these options, including linear regression replaces a pulse oximeter.

\section{FUTURE PROJECTS}

Develop different methodologies based on machine learning to more accurately estimate $\% \mathrm{SpO}_{2}$ with methodologies focused on signal analysis.

\section{ACKNOWLEDGMENTS}

To the "DESARROLLO DE UN SISTEMA MÓVIL-WEB COMO UNA SOLUCIÓN PREVENTIVA PARA EL REGISTRO Y SEGUIMIENTO DE PERSONAS QUE ESTÁN EN AISLAMIENTO DOMICILIARIO Y SON POSIBLES CASOS DE CORONA-VIRUS: APP COVID-LIFE” project, Wicon Energy investigate group and Universidad de las Fuerzas ArmadasESPE for funding.

\section{REFERENCES}

[1] T. Aoyagi. 1992. Pulse oximetry: Its origin and development. In 1992 14th Annual International Conference of the IEEE Engineering in Medicine and Biology Society, Vol. 7. 2858-2859.

[2] Solibella Bencomo, Sergio Villazana, and Bruno Salas. 2016. Design and construction of a pulse oximeter. http://servicio.bc.uc.edu.ve/ingenieria/ revista/v23n2/art06.pdf

[3] Organización Mundial de la Salud. 2020. Manejo clínico de la infección respiratoria aguda grave presuntamente causada por el nuevo coronavirus (2019-nCoV): orientaciones provisionales, 28 de enero de 2020. Technical documents. 11 p. pages. 
[4] Organización Mundial de la Salud. 2020. Preguntas y respuestas sobre la enfermedad por coronavirus (COVID-19). Technical Report. https: //www.who.int/es/emergencies/diseases/novel-coronavirus-2019/advice-for-public/q-a-coronaviruses\#: :text=sintomas

[5] X. Ding, D. Nassehi, and E. C. Larson. 2019. Measuring Oxygen Saturation With Smartphone Cameras Using Convolutional Neural Networks. IEEE Journal of Biomedical and Health Informatics 23, 6 (2019), 2603-2610. https://doi.org/10.1109/JBHI.2018.2887209

[6] J. García J. Martines. 2015. ¿Son útiles los dispositivos y aplicaciones inteligentes para la gestión de la salud? Comentario crítico. Enferm Cardiol 22 (08 2015), 33-37.

[7] Taylor B. Jordan, Cody L. Meyers, Walter A. Schrading, and John P. Donnelly. 2020. The utility of iPhone oximetry apps: A comparison with standard pulse oximetry measurement in the emergency department. The American Journal of Emergency Medicine 38, 5 (2020), 925-928. https: //doi.org/10.1016/j.ajem.2019.07.020

[8] A. K. Kanva, C. J. Sharma, and S. Deb. 2014. Determination of SpO2 and heart-rate using smartphone camera. , 237-241 pages.

[9] Luis Meza, Luis Enrique, and Silvia Ceballos. 2007. Design of procedures for the calibration of pulse-oximeters. https://dialnet.unirioja.es/descarga/ articulo/4787648.pdf

[10] P. M. Mohan, V. Nagarajan, and A. A. Nisha. 2017. A frame work to estimate heart rate and arterial oxygen saturation (Spo2). In 2017 International Conference on Communication and Signal Processing (ICCSP). 1645-1648. https://doi.org/10.1109/ICCSP.2017.8286669

[11] MSP 2020. Tableau

Public. https://public.tableau.com/profile/publicaciones.dneais.msp\#!/vizhome/COVID19ecu_MSP_15866333883550/COVID-19MSP

[12] Tara Parker. 2020. Why Days 5 to 10 Are So Important When You Have Coronavirus. https://www.nytimes.com/2020/04/30/well/live/coronavirus-days-5-through-10.html

[13] K. A. Reddy, B. George, N. M. Mohan, and V. J. Kumar. 2008. A Novel Method of Measurement of Oxygen Saturation in Arterial Blood. In 2008 IEEE Instrumentation and Measurement Technology Conference. 1627-1630. https://doi.org/10.1109/IMTC.2008.4547304

[14] K. A. Reddy, B. George, N. M. Mohan, and V. J. Kumar. 2009. A Novel Calibration-Free Method of Measurement of Oxygen Saturation in Arterial Blood. IEEE Transactions on Instrumentation and Measurement 58, 5 (2009), 1699-1705. https://doi.org/10.1109/TIM.2009.2012934

[15] Andrés Restrepo and Humberto Loaiza-Correa. 2012. Filtrado 3D espacio-temporal iterativo para la atenuación de ruido en secuencias de imágenes térmicas para END. Ingenium, Ciencia \& Tecnología 6 (09 2012), 27. https://doi.org/10.21774/ing.v6i13.85

[16] David Raimundo Rivas Lalaleo. 2019. Procesamiento digital de registros intracardíacos y de mapeo óptico. https://burjcdigital.urjc.es/handle/10115/ 16601

[17] H.and Martínez A. Rodríguez, J. A.and Garrido and M. A. García. 2013. Exactitud y Errores de la Oximetría de Pulso. In V Latin American Congress on Biomedical Engineering CLAIB 2011 May 16-21, 2011, Habana, Cuba, José Folgueras Méndez, Tania Y. Aznielle Rodríguez, Carlos F. Calderón Marín, Susana Beatriz Llanusa Ruiz, Jorge Castro Medina, Haddid Vega Vázquez, Maylen Carballo Barreda, and Rafael Rodríguez Rojas (Eds.). Springer Berlin Heidelberg, Berlin, Heidelberg, 758-761.

[18] José Luis Rojo-Álvarez, Manel Martínez-Ramón, Jordi Muñoz Marí, and Gustavo Camps-Valls. 2018. Digital signal processing with kernel methods. John Wiley Sons, Inc. 275 pages.

[19] C. G. Scully, J. Lee, J. Meyer, A. M. Gorbach, D. Granquist-Fraser, Y. Mendelson, and K. H. Chon. 2012. Physiological Parameter Monitoring from Optical Recordings With a Mobile Phone. IEEE Transactions on Biomedical Engineering 59, 2 (2012), $303-306$. https://doi.org/10.1109/TBME.2011.2163157

[20] usc.es. 2011. Regresion Lineal Multiple El modelo, estimación de los parámetros, contrastes. http://eio.usc.es/eipc1/BASE/BASEMASTER/ FORMULARIOS-PHP-DPTO/MATERIALES/Mat_50140128_RegresionMultiple.pdf 